\title{
Influence of Replicated Subsamples and Subsample Heterogeneity on the Linear Index of Food Selection ${ }^{1}$
}

\author{
Richard E. Strauss \\ Division of Fishes, Museum of Zoology \\ The University of Michigan, Ann Arbor, Michigan 48109
}

\begin{abstract}
In descriptive studies of prey preference, relative abundances of prey often are calculated from a series of replicated subsamples and total sample sizes are estimated rather than counted. When this is done, the apparent statistical power of the linear index of food selection $\left(L_{i}=r_{i}-p_{i}\right.$, where $r_{i}$ and $p_{i}$ are proportions of prey taxon $i$ in the gut and habitat, respectively) is artificially inflated. Additional biological variability, due either to environmental heterogeneity or to differences in feeding behavior among individual predators, will inflate further the apparent power of the index and also will produce underestimates of the degree of feeding selectivity. To correct for these problems, expressions that allow for subsampling designs and biological heterogeneity are provided for the standard error of the linear index and the associated degrees of freedom. The ratio of observed sampling variance in relative abundance to that expected for random sampling allows measurement of heterogeneity and statistical testing of the null hypothesis of homogeneity.
\end{abstract}

Several measures of prey selection have been proposed to compare the food eaten by predators and grazers with that potentially available (for example, Ivlev 1961; Frame 1974; Jacobs 1974; Chesson 1978; Cock 1978; Paloheimo 1979; Vanderploeg and Scavia 1979; Donaghay 1980). As with descriptive indices in general, each measure has its own sensitivities, biases, underlying assumptions, and technical inadequacies, each of which may vary in importance depending upon the particular experimental design.

Recently I proposed a generalized, linearly differential measure of food selection that is based on no specific model of behavior or ecological relationships but that possesses simple, interpretable mathematical properties and welldefined statistical behavior (Strauss 1979). The index was intended to supplant several similar but less reliable indices of preference commonly used in descriptive autecological studies. It is defined simply as

$$
L_{i}=r_{i}-p_{i}
$$

where $r_{i}$ is the relative abundance (proportion) of prey taxon $i$ in the gut of the predator, and

\footnotetext{
${ }^{1}$ Authorized for publication as Paper 6237 in the Journal Series of the Pennsylvania Agricultural Experiment Station, University Park, Pennsylvania 16802.
}

$p_{i}$ is the relative abundance of the same taxon in the environment. Relative abundance may be estimated by number, weight, or volume (Hyslop 1980).

Central to the interpretation of prey-selection data is a knowledge of the amount of sampling error to be expected. Point estimates of an index can be evaluated reasonably only in relation to associated confidence intervals, which reflect the degree of uncertainty of the estimates in relation to their range of possible values. Most indices of prey selection are complicated mathematical functions, and standard errors for them cannot be derived analytically. Estimates of the sampling variance, if evaluated at all, must be based on approximate variance expressions or on Monte Carlo simulations (Strauss 1979; Murtaugh 1981a). Such estimates indicate a general lack of precision, particularly within the range of sample sizes typical of most field studies. However, under a null hypothesis of random feeding in a homogeneous environment (and if the errors of measurement of $r_{i}$ and $p_{i}$ are statistically independent), the linear index is characterized by a small, predictable estimated sampling variance,

$$
\begin{aligned}
\mathrm{s}_{\text {exp }}^{2}\left(L_{i}\right) & =\mathrm{s}_{\text {exp }}^{2}\left(r_{i}\right)+\mathrm{s}_{\text {exp }}^{2}\left(p_{i}\right) \\
& =\frac{r_{i}\left(1-r_{i}\right)}{n_{r}}+\frac{p_{i}\left(1-p_{i}\right)}{n_{p}},
\end{aligned}
$$

where the binomial sample size $n_{r}$ is the total number of organisms of all taxa recovered from 
the guts of all predators examined, and $n_{p}$ is the total number of potential prey organisms collected from the habitat. Equation (2) can be used either to provide confidence intervals for an index value or to test whether or not two point estimates, or a point estimate and a nullhypothesis value, differ significantly with some assigned level of probability (for example, Hansen and Wahl 1981; Teska and Behmer 1981).

\section{Adjustment for Subsampling}

For prey samples of moderate size, Equation (2) provides an appropriate estimate of the expected sampling error. But when the numbers of prey organisms involved are very large, on the order of several thousand, sample sizes and relative abundances usually are estimated from replicated subsamples (Hutchinson 1971; Murtaugh 1981a). The intrinsic size variability among subsamples can increase the actual sampling error of the index over that estimated by Equation (2). When spurious degrees of freedom based on large estimated sample sizes then are applied to the standard error, the statistic can become so powerful that virtually any index value calculated will differ significantly from the null-hypothesis value for random feeding.

The apparent power of the linear index is artificially inflated for large sample sizes estimated from subsample counts. This results in part from the large associated degrees of freedom, and in part from use of $r_{i}, p_{i}, n_{r}$, and $n_{p}$ in the variance expression; these are estimates with associated sampling errors, rather than actual parameters of the populations. When the parameters of a population cannot be determined exactly, we face the compound problem of estimating the parameters from a sample or subsamples, assigning confidence limits to them, and then using the estimates to obtain a standard error for the index. Thus, the standard error itself has a variance component that is a function of the second and fourth moments of the component estimates (Kendall and Stuart 1977).

Normally, the error component of the standard error is small and can be ignored. But when the proportion of a prey item in the diet or habitat is estimated from a series of replicated subsamples, the second-order variance can be sizable and may lead to spurious conclusions about the significance of calculated index values. To correct for this we may apply the fol-

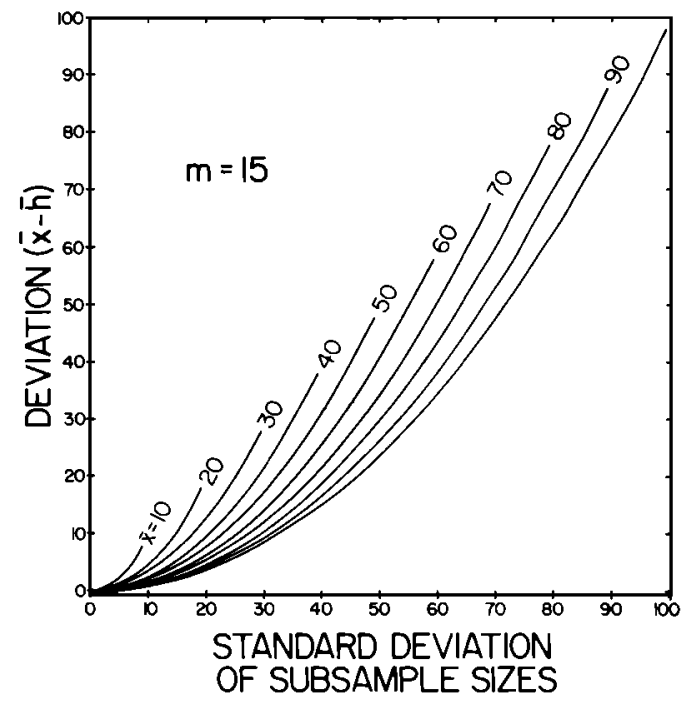

FIGURE 1.-Dependence of the harmonic mean sample size $(\overline{\mathrm{h}}$, expressed as a deviation from the arithmetic mean $\overline{\mathrm{x}}$ ) on the existing variation among subsample sizes, for the case of $\mathrm{m}=15$ subsamples. The standard deviation is used here as a measure of subsample-size variation to allow comparable axis scales; the deviation $\overline{\mathrm{x}}-\overline{\mathrm{h}}$ is linearly proportional to the variance of the subsample sizes.

lowing relationship: for $m$ subsamples of different sizes drawn from a common population in which the actual proportion of individuals of taxon $i$ is $T_{i}$ (estimated as $t_{i}$ ), the expected variance of the mean proportion of taxon $i$ in the subsamples is

$$
\mathrm{s}^{2}{ }_{\exp }\left(\bar{t}_{i}\right)=\frac{\bar{t}_{i}\left(1-\bar{t}_{i}\right)}{m \bar{h}}
$$

(Kendall and Stuart 1977). Here, $\bar{t}_{i}$ is the weighted mean proportion of taxon $i$ (weighted by subsample size and averaged across subsamples), and $\bar{h}$ is the harmonic mean subsample size. The harmonic mean is calculated as the reciprocal of the arithmetic mean of the reciprocals of the subsample sizes.

Use of the harmonic mean in Equation (3) in place of a single composite sample size increases the total variance of the expression so as to account exactly for the variance among the subsample sizes. If all subsamples contain the same number of prey items, use of Equation (3) will not change the standard error of the index over that estimated by Equation (2) because $m \bar{h}$ then equals $n$, the total sample size. However, if there 
exists variability among the subsample sizes, the harmonic mean will be proportionately less than the arithmetic mean (Fig. 1) and the standard error of the index will be correspondingly increased to reflect the influence of unequal subsampling on the reliability of the index value.

Application of Equation (3) to the expression for the sampling variance of $L_{i}$ is straightforward: it may be used to replace either the first or second term of Equation (2), or both. In the examination of gut contents, the gut of each predator (or collection of predators) would represent one of $m_{r}$ random subsamples, and $\bar{h}_{r}$ would be the harmonic mean of the numbers of prey organisms collected from the $m_{r}$ predators examined. The resulting variance of the mean proportion of prey taxon $i\left[\mathbf{s}_{\mathbf{e x p}}^{2}\left(\bar{t}_{i}\right)\right]$ then would replace the first term $\left[\mathrm{s}^{2}{ }_{\exp }\left(r_{i}\right)\right]$ in Equation (2). In the case of $m_{p}$ habitat subsamples, $\bar{h}_{p}$ is the harmonic mean number of potential prey organisms in each subsample and $s^{2}{ }_{\text {exp }}\left(\bar{t}_{i}\right)$ replaces the second term $\left[\mathrm{s}^{2}{ }_{\exp }\left(p_{i}\right)\right]$ of Equation (2).

The resulting estimate of the standard error of the index (the square root of the sampling variance) and the associated degrees of freedom together determine the statistical significance of a particular index value. Total degrees of freedom are the number of terms contributing to the variance minus the number of constants required to calculate the expected value of $L_{i}$. When $n_{r}$ and $n_{p}$ are counts from which $r_{i}$ and $p_{i}$ are derived (Equation 2), there are $n_{r}+n_{p}$ terms contributing to the variance and two constants $\left(n_{r}\right.$ and $\left.n_{p}\right)$ required for the expected value; total degrees of freedom are thus $n_{r}+n_{p}-2$ (Strauss 1979). However, when $m_{r}$ (from the predators' guts) or $m_{p}$ (from the habitat) subsamples are collected and used to estimate $\bar{r}_{i}$ or $\bar{p}_{i}$ (by means of Equation 3), there are $m_{r} \bar{x}_{r}$ terms (where $\bar{x}_{r}$ is the arithmetic mean of the sizes of the subsamples taken from the predators' guts) contributing to the estimated variance of $\bar{r}_{i}$, and $m_{p} \bar{x}_{p}$ terms to that of $\bar{p}_{i}$. The associated degrees of freedom for the components of $\mathrm{s}\left(L_{i}\right)$ are thus $m_{r} \bar{x}_{r}-1$ and $m_{p} \bar{x}_{p}-1$ when both the diet and habitat are subsampled. Total degrees of freedom are $m_{r} \bar{x}_{r}+m_{p} \bar{x}_{p}-2$. With a mixed experimental design in which either the diet or the habitat is subsampled, but not both, the appropriate degrees of freedom for $\mathrm{s}\left(L_{i}\right)$ would be either $m_{r} \bar{x}_{r}+n_{p}-2$ or $n_{r}+$ $m_{p} \bar{x}_{p}-2$ respectively.

\section{Measurement and Adjustment for Heterogeneity}

The standard error adjusted for subsampling accounts only for the additional uncertainty in the index resulting from random variability in a homogeneous environment. If additional unrecognized variability is present, due either to systematic variation in predator behavior (as among different sexes, sizes, or age classes) or to heterogeneity in the environment, then a composite index value will tend to underestimate the degree of prey selection by individual predators or in particular habitats. Systematic variation also will cause index values to be less reliable than expected. A well-designed study of prey selection will take into account the known heterogeneity in the predator or habitat (Murtaugh 1981b). However, the observed variance in relative abundances among subsamples may be used both to test for unrecognized heterogeneity and to compensate for it by systematically increasing the widths of confidence intervals.

Systematic variation will tend to increase the observed variance in relative abundance over that expected from random sampling in a homogeneous environment. This suggests use of the ratios of the two variances $\left(\mathrm{s}^{2}{ }_{\mathrm{obs}} / \mathrm{s}^{2}\right.$ exp $)$ as measures of heterogeneity:

$$
\begin{aligned}
& H_{r}=\frac{\mathrm{s}^{2}{ }_{\mathrm{obs}}\left(r_{i}\right)}{\mathrm{s}_{\text {exp }}^{2}\left(r_{i}\right)}=\frac{\mathbf{s}^{2}{ }_{\mathrm{obs}}\left(r_{i}\right) \cdot m_{r} \bar{h}_{r}}{\bar{r}_{i}\left(1-\bar{r}_{i}\right)} ; \\
& H_{p}=\frac{\mathrm{s}^{2}{ }_{\mathrm{obs}}\left(p_{i}\right)}{\mathrm{s}^{2}{ }_{\mathrm{exp}}\left(p_{i}\right)}=\frac{\mathrm{s}^{2}{ }_{\mathrm{obs}}\left(p_{i}\right) \cdot m_{p} \bar{h}_{p}}{\bar{p}_{i}\left(1-\bar{p}_{i}\right)} ;
\end{aligned}
$$

where $H_{r}$ is the estimated heterogeneity among individual predators, $\mathrm{s}_{\text {obs }}{ }\left(r_{i}\right)$ is the observed weighted variance (weighted by subsample size) among the $m_{r}$ predators, $H_{p}$ and $\mathrm{s}^{2}{ }_{\mathrm{obs}}\left(p_{i}\right)$ are corresponding estimates for habitat subsamples, and the other variables are as defined above. The expected values of both ratios are 1.0 for a homogeneous system. Although the ratios $H_{r}$ and $H_{p}$ may be regarded simply as descriptive measures, they also may be used as test statistics; when the "population" variances $\left(\sigma^{2}{ }_{\text {obs }}, \sigma^{2}\right.$ exp $)$ are equal, the ratio of the two sample variances follows an $F$-distribution with 2 and $(m \bar{x}-1) \mathrm{df}$, allowing a direct test of the null hypothesis of homogeneity. Calculated ratio values may vary from zero to infinity. Values greater than 3 allow rejection of the null hypothesis of homogeneity at a significance level of approximately $P=0.05$. 
TABLE 1.-Hypothetical data for the relative occurrences and corresponding selection-index values for a single prey taxon, in six $\left(=\mathrm{m}_{\mathrm{p}}\right)$ random habitat subsamples and six $\left(=\mathrm{m}_{\mathrm{r}}\right)$ randomly selected predators representing two age classes. Given are the total number of prey collected per habitat subsample $\left(\mathrm{n}_{\mathrm{pk}}\right)$ and proportions of prey taxon 1 ( $\mathrm{p}_{1 \mathrm{k}}$ ) in each; the total number of prey collected per predator $\left(\mathrm{n}_{\mathrm{ri}}\right)$ and the proportions of taxon 1 in each $\left(\mathrm{r}_{1 \mathrm{j}}\right)$; and the selection-index values $\left(L_{1 \mathrm{j}}\right)$ and associated standard errors for each predator.

\begin{tabular}{cccccccc}
\hline $\begin{array}{c}\text { Habitat } \\
\text { subsample } \\
(k)\end{array}$ & $n_{p k}$ & $p_{1 k}$ & $\begin{array}{c}\text { Predator } \\
(j)\end{array}$ & Age class & $n_{r j}$ & $r_{\mathbf{1 j}}$ & $\mathrm{L}_{\mathbf{1 j}} \pm \mathrm{SE}_{\mathbf{e x p}}\left(L_{\mathbf{1 j}}\right)$ \\
\hline 1 & 55 & 0.43 & 1 & I & 25 & 0.30 & $-0.15 \pm 0.10$ \\
2 & 74 & 0.46 & 2 & I & 28 & 0.34 & $-0.11 \pm 0.10$ \\
3 & 60 & 0.37 & 3 & I & 34 & 0.32 & $-0.13 \pm 0.09$ \\
4 & 66 & 0.52 & 4 & II & 16 & 0.71 & $0.26 \pm 0.12$ \\
5 & 60 & 0.44 & 5 & II & 20 & 0.75 & $0.30 \pm 0.11$ \\
6 & 64 & 0.48 & 6 & II & 21 & 0.73 & $0.28 \pm 0.11$ \\
\hline
\end{tabular}

As an example, consider a case in which the gut contents of six individual fishes, representing two age classes with very different food preferences, are to be compared with six random subsamples of prey (such as Surber samples) taken from a slightly heterogeneous habitat. The gut contents of each fish represent one of six $\left(=m_{r}\right)$ subsamples of the diet, while each habitat subsample represents one of six $\left(=m_{p}\right)$ subsamples of the environment (Table 1). In this example the observed weighted variance of relative prey proportions among the habitat subsamples is somewhat greater than that expected from a homogeneous environment, resulting in a marginally significant heterogeneity estimate of $H_{p}=3.2(P=0.04$; Table 2$)$. The corresponding estimate of $H_{r}=27.6$ among the six predators reveals a highly significant variability in feeding selectivity $(P<0.0001)$ which in this case is directly attributable to age-class differences. The separate heterogeneity estimates for the two age classes are both near zero
( $H_{r}=0.097$ and $H_{p}=0.073$, respectively), and selection-index values for the two groups are significantly different $\left(L_{I}=-0.14 \pm 0.06\right.$ and $L_{I I}=0.28 \pm 0.06$ ).

This example is simplistic. Such age-specific differences in feeding behavior should be recognized, or at least suspected, long before the calculations are performed. However, effects of behavioral or environmental heterogeneity may be much more subtle and may not be attributable to any obvious biological phenomenon. In such cases average selection-index values may have to be reported and additional unreliability due to heterogeneity must be represented by increased confidence-interval widths. The adjusted standard error should reflect both the observed variability in relative abundances among subsamples and the average uncertainty of any particular relative-abundance estimate. It may be calculated as follows.

(1) Estimate the relative occurrence, and its sampling variance, of prey taxon $i$ in the envi-

TABLE 2.-Sampling-variance and heterogeneity calculations, based on relative-occurrence data of Table 1, for the habitat subsamples and for gut contents of the predators, both pooled and considered separately by age class. $\sigma^{2}$ is "population" variance.

\begin{tabular}{|c|c|c|c|c|c|}
\hline \multicolumn{2}{|l|}{ Parameter } & \multirow{2}{*}{$\begin{array}{c}\text { Habitat } \\
\text { subsamples }\end{array}$} & \multirow{2}{*}{$\begin{array}{c}\text { Gut- } \\
\text { content } \\
\text { samples }\end{array}$} & \multicolumn{2}{|c|}{ Predator age classes } \\
\hline Description & Notation & & & I & II \\
\hline Number of subsamples & $m_{p}$ or $m_{r}$ & 6 & 6 & 3 & 3 \\
\hline Total sample size & $n_{p}$ ог $n_{\tau}$ & 379 & 144 & 87 & 57 \\
\hline Mean subsample size & $\bar{x}_{p}$ or $\bar{x}_{r}$ & 63.2 & 24.0 & 29.0 & 19.0 \\
\hline Harmonic mean subsample size & $\hat{h}_{p}$ or $\bar{h}_{r}$ & 62.6 & 22.6 & 28.5 & 18.7 \\
\hline Mean relative occurrence & $\bar{r}_{1}$ or $\bar{p}_{1}$ & 0.452 & 0.483 & 0.320 & 0.731 \\
\hline Expected sampling variance & $s_{\exp }^{2}$ & 0.0007 & 0.0018 & 0.0025 & 0.0035 \\
\hline Observed sampling variance & $\mathrm{s}^{2}{ }_{\mathrm{obs}}$ & $0.002 \mathrm{I}$ & 0.0409 & 0.0003 & 0.0003 \\
\hline Heterogeneity & $H_{p}$ or $H_{r}$ & 3.202 & 27.58 & 0.097 & 0.073 \\
\hline Probability of homogeneity & $P\left(\sigma^{2}{ }_{\mathrm{obs}}=\sigma^{2}{ }_{\text {exp }}\right)$ & 0.04 & $<0.0001$ & 0.99 & 0.93 \\
\hline
\end{tabular}


ronment. For a single habitat sample, these are simply $p_{i}$ and $s^{2}$ exp $\left(p_{i}\right)$ from Equations (1) and (2). For subsamples, the best estimate of relative abundance is the weighted mean $\bar{p}_{i}$ (Equation 3) and a reasonable estimate of the sampling variance is the observed variance among subsamples plus the mean expected variance of the individual subsample estimates:

$$
\mathrm{s}^{2}\left(\bar{p}_{i}\right)=\mathrm{s}_{\mathrm{obs}}^{2}\left(p_{i}\right)+\frac{1}{m_{p}} \sum_{k=1}^{m_{p}} \frac{p_{i k}\left(1-p_{i k}\right)}{n_{p k}} .
$$

(2) In the same way, estimate the relative occurrence and associated sampling variance for prey $i$ in the diet of predator $j$. These are $r_{i}$ and $\mathrm{s}^{2}{ }_{\text {exp }}\left(r_{i}\right)$ for a single sample or, for subsamples are the weighted mean $\bar{r}_{i}$ and

$$
\mathrm{s}^{2}\left(\bar{r}_{i}\right)=\mathrm{s}_{\text {obs }}^{2}\left(r_{i}\right)+\frac{1}{m_{r}} \sum_{j=1}^{m_{r}} \frac{r_{i j}\left(1-r_{i j}\right)}{n_{r j}} .
$$

(3) The composite preference index and its sampling variance are then simply

$$
L_{i}=\bar{r}_{i}-\bar{p}_{i}
$$

and

$$
\mathrm{s}^{2}\left(L_{i}\right)=\mathrm{s}^{2}\left(\bar{r}_{i}\right)+\mathrm{s}^{2}\left(\bar{p}_{i}\right) .
$$

The adjusted standard error [the square root of $\left.\mathrm{s}^{2}\left(L_{i}\right)\right]$ accounts in an approximate way for the effects of both subsampling and estimated heterogeneity. Because the data used to estimate heterogeneity are the same as those accounting for the effects of subsampling, no information has been lost or gained and degrees of freedom are unaffected.

\section{Discussion}

The general utility of the linear index is due to its simple mathematical structure and statistical reliability, which should reflect the chosen sampling design. The assignment of confidence limits to this or any other index is important in view of the biological inferences that often are drawn from differences in their values. Strauss (1979) discussed the calculation of a standard error for the prey-preference index $L_{i}$ when the prey populations and numbers eaten are small enough to be directly counted. The present paper provides modified expressions for the standard error given the need for subsampling and the likely presence of some biological heterogeneity in the system.

The adjusted standard error will account for the additional uncertainty in the index value resulting from variation among individual predators or among different subsamples of the predator's habitat. This is true whether the variability results from random fluctuations in sampled prey abundances or from systematic variation in predator behavior or spatial distribution of prey. Thus, unlike most other indices, the corrected linear index and its standard error do not assume a homogeneous environment, and in fact assume no particular spatial distribution. Although a well-designed study of prey selection will take account of known systematic variability in the environment or predator, the adjusted standard error will compensate for any unrecognized heterogeneity by increasing the vidths of confidence intervals. In addition, Equations (4) and (5) allow estimates of, and statistical tests for, such heterogeneity.

\section{Acknowledgments}

I thank M. A. Houck, E. L. Cooper, P. A. Murtaugh, D. Scavia, R. A. J. Taylor, and F. M. Williams for their constructive comments on the manuscript, and W. Burton for first bringing this problem to my attention. This work was supported by the Pennsylvania Agricultural Experiment Station and by National Science Foundation Grants DEB-7903285 and DEB8011562.

\section{References}

Chesson, J. 1978. Measuring preference in selective predation. Ecology 59:2 11-215.

Cock, M. J. W. 1978. The assessment of preference. Journal of Animal Ecology 47:805-816.

Donaghay, P. L. 1980. Experimental and conceptual approaches to understanding algal-grazer interactions. Doctoral dissertation. Oregon State University, Corvallis, Oregon, USA.

Frame, D. W. 1974. Feeding habits of young winter flounder (Pseudopleuronectes americanus): prey availability and diversity. Transactions of the American Fisheries Society 103:261-269.

Hansen, M. J., and D. H. WAHL. 1981. Selection of small Daphnia pulex by yellow perch fry in Oneida Lake, New York. Transactions of the American Fisheries Society 110:64-71.

Hutchinson, B. P. 1971. The effect of fish predation on the zooplankton of ten Adirondack lakes, with particular reference to the alewife, Alosa pseudoharengus. Transactions of the American Fisheries Society 100:325-335.

Hyslop, E. J. 1980. Stomach contents analysis-a review of methods and their application. Journal of Fish Biology 17:411-429.

IVleV, V. S. 1961. Experimental ecology of the feed- 
ing of fishes. Yale University Press, New Haven, Connecticut, USA.

JACOBS, J. 1974. Quantitative measurement of food selection: a modification of the forage ratio and Ivlev's electivity index. Oecologia (Berlin) 14:413417.

Kendall, M., and A. Stuart. 1977. The advanced theory of statistics, volume 1 , 4th edition. Macmillan, New York, New York, USA

Murtaugh, P. A. 198 la. Selective predation by $\mathrm{NeO}-$ mysis mercedis in Lake Washington. Limnology and Oceanography 26:445-453.

Murtaugh, P. A. 1981 b. Size-selective predation on Daphnia by Neomysis mercedis. Ecology 62:894-900.
Paloheimo, J. E. 1979. Indices of food type preference by a predator. Journal of the Fisheries Research Board of Canada 36:470-473.

STrauss, R. E. 1979. Reliability estimates for Ivlev's electivity index, the forage ratio, and a proposed linear index of food selection. Transactions of the American Fisheries Society 108:344-352.

Teska, J. D., AND D. J. Behmer. 1981. Zooplankton preference of larval lake whitefish. Transactions of the American Fisheries Society 110:459-461.

Vanderploeg, H. A., And D. Scavia. 1979. Two electivity indices for feeding with special reference to zooplankton grazing. Journal of the Fisheries Research Board of Canada 36:362-365. 